\title{
Reply to comment on Agarwal et al.: Does initial Pirani score and age influence number of Ponseti casts in children?
}

\author{
Uday Guled • Nirmal Raj Gopinathan • Vijay Goni • \\ Venkata Ramana
}

Received: 29 May 2014 / Accepted: 2 July 2014 / Published online: 16 July 2014

(C) SICOT aisbl 2014

We read the reply by the authors of the article by Agarwal et al. [1]. We congratulate them for a quick reply for an important issue raised in the article. We also agree that the resistant and recurrent clubfoot are a special category of clubfoot patients which require an individualized approach. It has been repeatedly proven that parental education status affects the compliance and hence the prognosis of clubfoot [2], and the authors are in agreement with us.

We still stick to our opinion that assessment of severity of clubfoot and conservative management of clubfoot in older children is quite different. Assessment of severity by Pirani score is difficult since there are no empty heel signs, distinct medial and posterior creases in walking children [3]. We still feel that it is necessary to modify the Ponseti technique of conservative management in neglected clubfoot, including the duration of manipulation, interval between cast change, maximum abduction limit, indication for tenotomy, and post correction brace protocol. Though this issue has been raised by many articles [3-5], the topic remains controversial. We appreciate the present authors effort in ad- dressing an important issue in the management of clubfoot.

\section{References}

1. Agarwal A, Gupta N (2004) Does initial Pirani score and age influence number of Ponseti casts in children? Int Orthop 38:569-572. doi:10. 1007/s00264-013-2155-3 (2014)

2. Dobbs MB et al (2004) Factors predictive of outcome after use of the Ponseti method for the treatment of idiopathic clubfeet. J Bone Joint Surg 86-A:22-27

3. Spiegel DA et al (2009) Ponseti method for untreated idiopathic clubfeet in Nepalese patients from 1 to 6 years of age. Clin Orthop Relat Res 467:1164-1170. doi:10.1007/s11999-008-0600-1

4. Khan SA, Kumar A (2010) Ponseti's manipulation in neglected clubfoot in children more than 7 years of age: a prospective evaluation of 25 feet with long-term follow-up. J Pediatr Orthop 19:385-389. doi: 10.1097/BPB.0b013e3283387cc8

5. Wang YZ, Wang XW, Zhang P, Wang XS (2009) Application of Ponseti method in patients older than 6 months with congenital talipes equinovarus. Beijing da xue xue bao Yi xue ban=J Peking Univ Health Sci 41:452-455

U. Guled $(\bowtie) \cdot$ N. R. Gopinathan $\cdot$ V. Goni $•$ V. Ramana Department of Orthopaedics, PGIMER, Chandigarh, India e-mail: drudayguled@gmail.com 